\title{
DIAGNÓSTICO DA IMPLANTAÇÃO DE Eucalyptus grandis E Syzygium jambos NO CAMPUS DA UNIVERSIDADE FEDERAL DE PERNAMBUCO, ATRAVÉS RELAÇÃO DA IDADE COM O VOLUME DE MADEIRA
}

Danilo Quintas Souto Maior ${ }^{1}$, Otacilio Antunes Santana ${ }^{1}$, Euzelina Borges dos Santos Inácio ${ }^{1}$, Laerte Bezerra de Amorim $^{2}$, José Imaña Encinas ${ }^{3} 4$

\section{RESUMO}

O objetivo deste trabalho foi avaliar duas espécies arbóreas (Eucalyptus grandis e Syzygium jambos) implementadas no Campus Universitário da Universidade Federal de Pernambuco/Campus Recife, no final da década de 40 (Século XX), quanto a sua relação idade e volume; e discutir a implementação destas espécies no plano de arborização do campus. As idades foram mensuradas através dos anéis de crescimentos, coletados com trado de incremento. Foram utilizados 40 indivíduos e analisados um período de 10 anos (2001-2010). O volume das espécies foi determinado por meio da relação entre o volume real do tronco pelo volume do cilindro. Os resultados mostram que a variável volume possui uma relação diretamente proporcional e significativa com a variável idade, sendo assim os indivíduos da espécie Eucalyptus grandis apresentaram um crescimento do tronco maior que Syzygium jambos, podendo ser devido ao modo de investimento de seus produtos fotossintéticos, conforme discutidos pela literatura. O crescimento dos indivíduos arbóreos no planejamento do Campus da Universidade Federal de Pernambuco atenderam as expectativas propostas, porém, com a expansão das vias e das construções, os indivíduos arbóreos tornaram riscos a danos a estas instalações.

Palavras-chave: dendrocronologia; áreas urbanas; espécies exóticas.

\section{DIAGNOSIS OF IMPLEMENTATION OF Eucalyptus grandis E Syzygium jambos AT FEDERAL UNIVERSITY OF PERNAMBUCO CAMPUS, THROUGH RELATIONSHIP OF AGE WITH THE WOOD VOLUME}

\begin{abstract}
The objective of this work was to evaluate two tree species (Eucalyptus grandis and Syzygium jambos) implemented at the Campus of Federal University of Pernambuco / Recife Campus in the late 40s (Twentieth Century), by the parameters were age and volume, and to discuss the implementation of these species in the Campus planning and actual management. Ages were measured through growth rings, collected with increment borer. A total of 40 individuals were analyzed a period of 10 years (2001-2010). The volume of species was determined by the relationship between wood volume by cylinder volume. The results show that the variable volume has a significant and directly proportional to the age variable, so the individuals of the species Eucalyptus grandis grew larger the wood than Syzygium jambos, which may be due to their investment model of photosynthetic products as discussed in the literature. The growth of trees individual in the planning of Federal University of Pernambuco got expectations proposals, however, with the expansion of roads and buildings, individual trees become risks damage to these instalations.
\end{abstract}

Key words: dendrochronology; urban areas; exotic species.

\footnotetext{
${ }_{1}^{1}$ Departamento Biofísica e Radiobiologia, Centro de Ciências Biológicas, Universidade Federal de Pernambuco. danilo_quintas@hotmail.com; otacilio.santana, euzelina.inacio[ufpe.br].

${ }^{2}$ Departamento de Energia Nuclear, Centro de Tecnologia e Geociências, Universidade Federal de Pernambuco. laerteamorim@yahoo.com.br

${ }^{3}$ Departamento de Engenharia Florestal, Faculdade de Tecnologia, Universidade de Brasília. imana@unb.br

${ }^{4}$ recebido em 24.05.2012 e aceito para publicação em 15.06.2013
} 
Os indivíduos arbóreos introduzidos, pertencentes a espécies exóticas, são classificados como aqueles que estão em outros habitats que não são o de sua origem, tendo alta taxa de dispersão e crescimento, e com isso competindo diretamente por luz e nutrientes com as espécies nativas (PIECK et al., 2011). No Brasil, várias espécies exóticas foram introduzidas, não só por um caráter de produção, como no caso das monoculturas, mas também as de caráter urbanísticos e de produção doméstica. Duas destas espécies são Eucalyptus grandis Hill ex. Maiden (Myrtaceae) e Syzygium jambos L. (Myrtaceae), popularmente denominados de eucalipto-grandis e jambeiro.

O Eucalyptus grandis é a espécie florestal mais plantada no Brasil (SOUZA et al., 2004), devido ao seu potencial produtivo e às características da madeira. Esta é utilizada para produção de celulose e papel, painéis de fibra e aglomerado, combustível industrial e doméstico e produtos de serraria, sendo a espécie nativa da costa leste da Austrália (SOARES et al., 2003). Também pertencente à família Myrtaceae, Syzygium jambos é uma árvore de origem asiática, nativa da Índia e Malásia. Seus frutos são édulos, chamativos pela cor entre o violeta e o vermelho, apreciados pelo aroma, atraindo a fauna e insetos (PIO CORREA, 1969), e empregados pelos humanos como diuréticos, digestivos e antifebris (CRAVO, 1994). As folhas são empregadas na medicina popular como digestivas e anti-inflamatórias (SLOWING et al., 1996).

O fator tempo é uma importante variável nestas espécies, pois como foram introduzidas aqui no Brasil a literatura as descreve com um alto poder de dispersão das sementes e crescimento de sua parte aérea, tronco e copa (ARIAS et al., 2011; ENCINAS et al., 2005). Ao passar do tempo os indivíduos destas espécies apresentam alto valor de importância fitossociológica no contexto local, havendo altos valores de densidade, dominância e frequência de seus indivíduos (MILLWARD; SABIR, 2011; RAMÍREZ et al., 2011). Com isso o valor do volume total por área $\left(\mathrm{m}^{3} / \mathrm{ha}\right)$ aumenta.

A relação idade/volume foi descrita em vários trabalhos da literatura, destacando: a competição por luz e nutrientes (BACILIO et al., 2011); indicadores de mudanças climáticas (BOULILA et al., 2011); ciclagem de nutrientes (BROWN et al., 2011); produção de serapilheira (COUTURIER et al., 2011); salinidade no solo (FEIKEMA; BAKER, 2011); eficiência na produção de frutos (HUTTON; LOVEYS, 2011); produção de madeira (HYNYNEN et al., 2011); amortizadores das mudanças climáticas (KANTARC et al., 2011); diferenças de altitude e pressão (KRUMM et al., 2011); mortalidade da espécie (MEYER; SCHMIDT, 2011); decomposição microbiana (PRESTON et al., 2011); distrofismo do solo (SINGH et al., 2011); concentração de princípios ativos (SREELATHA et al., 2011); efeitos da herbivoria (STRAW et al., 2011); planejamento urbano (VALDEZ-AGUILAR; CARTMILL, 2011); acúmulo de carbono (WANG et al., 2011); indicadores geomorfológicos (YOSSIFOVA et al., 2011); e outros.

Atualmente, o estudo do volume de madeira é de importante valia, pois além de fornecerem valores de madeira que serão utilizadas na produção de celulose, móveis, direcionados a construção civil, lenha e outros, está sendo utilizado nos cálculos de acúmulo de carbono, fator para redução dos níveis

Danilo Quintas Souto Maior et al. 
de $\mathrm{CO}_{2}$ presentes na atmosfera e importante para o impedimento do aumento do aquecimento global (WU et al., 2011). A atividade de acúmulo de carbono se dá pelo processo de fotossíntese em que as plantas através do $\mathrm{CO}_{2}$ e outras moléculas, produzem carboidrato, que são bioacumulados em seus tecidos vegetais de sustentação e investidos em outros tecidos como frutos, sementes, folhas, e outros (CARNEIRO et al., 2011). A cada ciclo este incremento de tecido vegetal forma na anatomia do caule dos indivíduos arbóreos, zonas de crescimentos anuais denominados anéis de crescimento, visíveis em algumas espécies, na qual é possível verificar a datação de cada indivíduo e observar períodos de variação do incremento seguindo respostas ambientais como a sazonalidade do regime de chuvas e de temperatura (FRANZARING et al., 2011).

O valor biométrico de um determinado tecido vegetal é particular de espécie para espécie (CARNEIRO et al., 2011). Algumas espécies, por exemplo, o Syzygium, desenvolve o fruto vistoso (epicarpo de intensa coloração) e um mesocarpo atrativo (altas quantidades de aminoácidos), para atração e disseminação da semente pela fauna, tendo assim maior possibilidade de sucesso no nascimento da semente filha (PIO CORREA, 1969). Por outro lado, as espécies de Eucalyptus, investem seus produtos metabólitos em folhas com substâncias alelopáticas, na qual forma uma serapilheira tóxicas, dificultando o crescimento de indivíduos de outras espécies vegetais, isto tudo para não haver competição por luz e nutrientes em suas adjacências (SOUZA et al., 2004).

No planejamento urbano quando estas espécies são utilizadas vários parâmetros antes da utilização das espécies arbóreas são avaliados (SEMENZATO et al., 2011). Parâmetros positivos (JIM; ZHANG,
2013) como: i) o conforto térmico para proporcionar um sombreamento, e ii) se serão utilizados para outros fins como quebra vento; e parâmetros negativos (MOLLER; SKOU; KOLLMANN, 2012) como: i) a expansão de galhos sob a rede elétrica, ii) a expansão das raízes sob calçamentos, iii) a dificuldade de manejo pela altura, e iv) atração de insetos e faunas devido aos frutos ou gomas são mensurados e ponderados no momento do plantio. Porém para um campus universitário estas espécies poderiam se encaixar em seu plano urbanístico devido aos espaços verdes entre as construções. Segundo a Prefeitura da Cidade Universitária da Universidade Federal de Pernambuco (UFPE/Campus Recife - PCU, 2103), os indivíduos de Eucalyptus grandis foram introduzidos no Campus como opções de quebravento e sombreamento para espaços verdes que circundavam construções que requeriam redução de ruídos ventos, como a biblioteca e de laboratórios centrais; e Syzygium jambos foram introduzidos para sombreamento, beleza cênica e disposição em adjacências a caminhos de pedestre entre as construções, isto tudo no final da década de 40 do século XX (1948).O objetivo geral foi avaliar duas espécies arbóreas (Eucalyptus grandis e Syzygium jambos) implementadas no Campus Universitário da UFPE, no final da década de 40 (Século XX), quanto a sua relação idade e volume; e discutir a implementação destas espécies no plano de arborização do campus. Os objetivos específicos deste trabalho foram: i) mensurar a altura, o diâmetro e os anéis de crescimento de indivíduos arbóreos de Eucalyptus grandis e Syzygium jambos; ii) mensurar os anéis de crescimento e determinar a idade destes indivíduos; iii) calcular o volume do tronco destes indivíduos; iv) analisar durante o período de 2001 a 2010 o incremento médio anual 
das espécies dos anéis de crescimento; v) relacionar a idade dos indivíduos em 2011 com os respectivos volumes de tronco; e vi) analisar e recomendar os indivíduos arbóreos das espécies estudadas no Campus da UFPE/Recife atualmente.

\section{MATERIAIS E MÉTODOS}

A área de estudo foi o campus da Universidade Federal de Pernambuco, Recife-PE (Figura 1), onde foram mensurados 80 indivíduos arbóreos, sendo 40 da espécie Eucalyptus grandis Hill ex. Maiden (Myrtaceae) e 40 da espécie Syzygium jambos L. (Myrtaceae). Para determinação da idade de todos indivíduos arbóreos foram coletadas amostras do tronco com um trado de incremento (Haglöf Sweden AB), a 1,3 m de altura, para análise dos anéis de crescimentos (Figura 2). Após a coleta a amostra de madeira foi enviada ao laboratório onde foi desumidificada a uma temperatura ambiente e quantificados e mensurados o tamanho dos anéis de crescimentos através de um paquímetro digital com precisão de graduação $0,02 \mathrm{~mm} / 0,001$ e de um microscópio Binocular 1000X LM3000B (UFNALSKI, 2005). A datação seguiu o método de “crossdating" entre os indivíduos, certificando o período para posterior análise (MAXWELL et al., 2011). O período para análise foi de 2001 a 2010 (10 anos), período em que todos os indivíduos existiam.

Figura 1. Localização da área de estudo e as espécies A) Eucalyptus grandis e B) Syzygium jambos no Campus da Universidade Federal de Pernambuco/Recife.

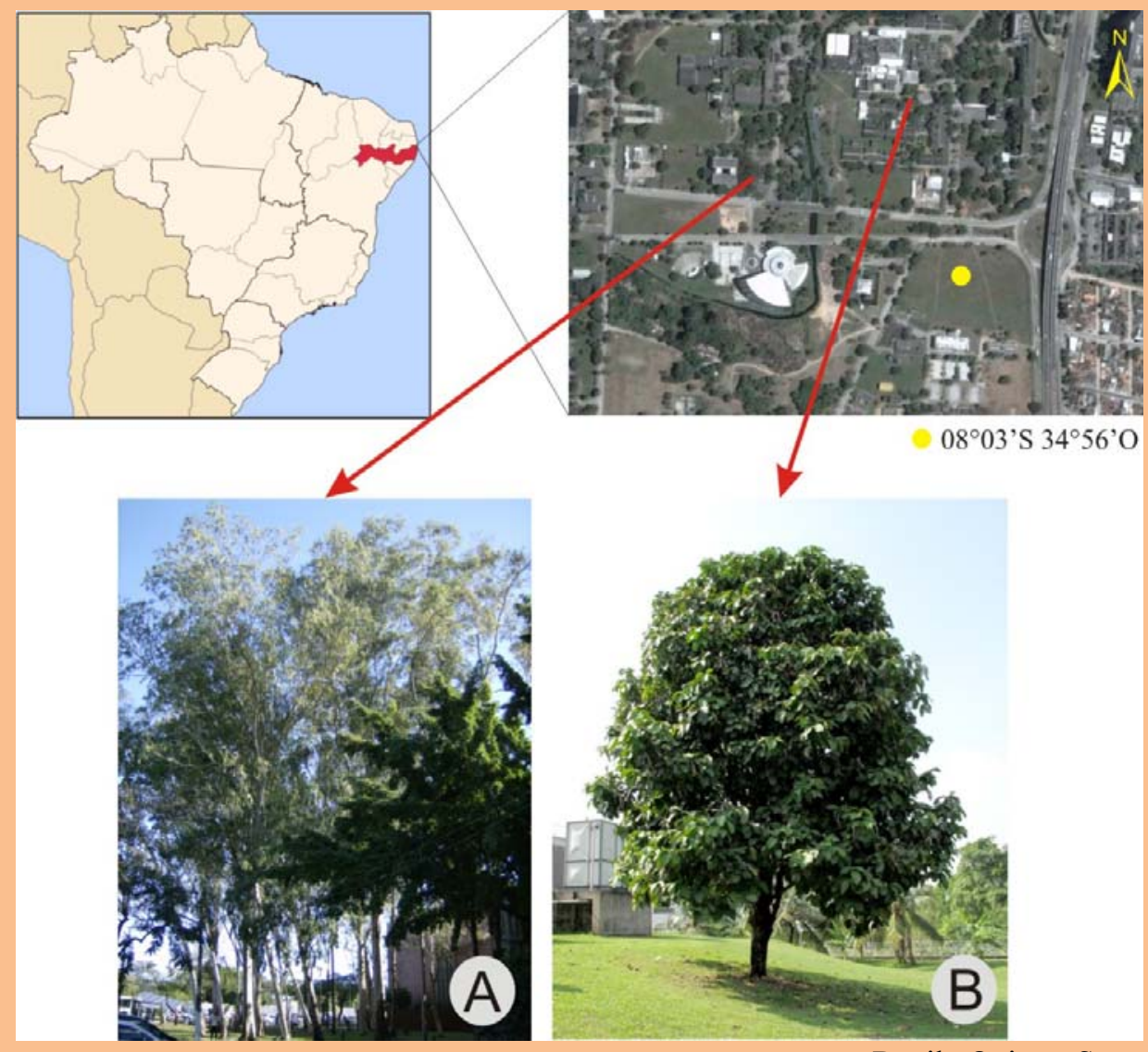

Danilo Quintas Souto Maior et al. 
Figura 2. A) Amostragem de coleta do incremento de madeira; B) Incremento de madeira saindo do equipamento de coleta com os anéis de crescimento.

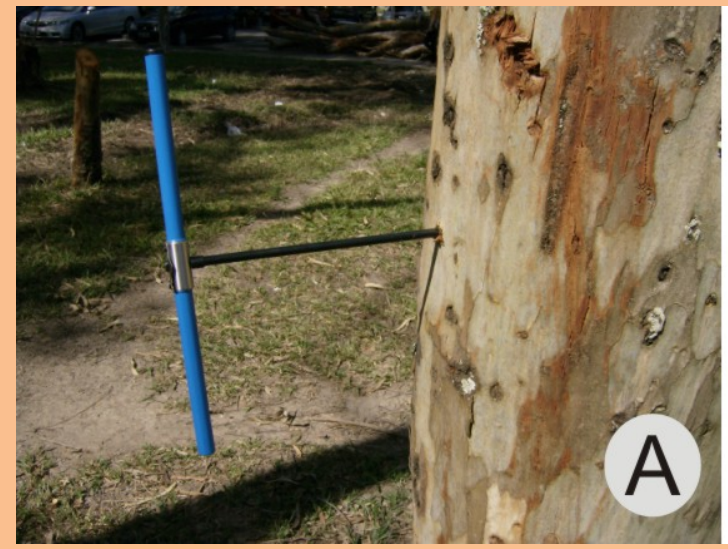

O diâmetro a altura do peito $(1,30 \mathrm{~m})$ foi mensurado com uma trena e a altura pelo método de referência de altura (Figura 3). Estas duas variáveis foram mensuradas (Agosto de 2011) para o cálculo do volume do tronco arbóreo que foi determinado através da equação (Eq. 1) (ZHOU et al., 2011):

$$
V o l=\pi \cdot\left(\frac{d}{2}\right)^{2} \cdot h \cdot f f
$$

Eq.1

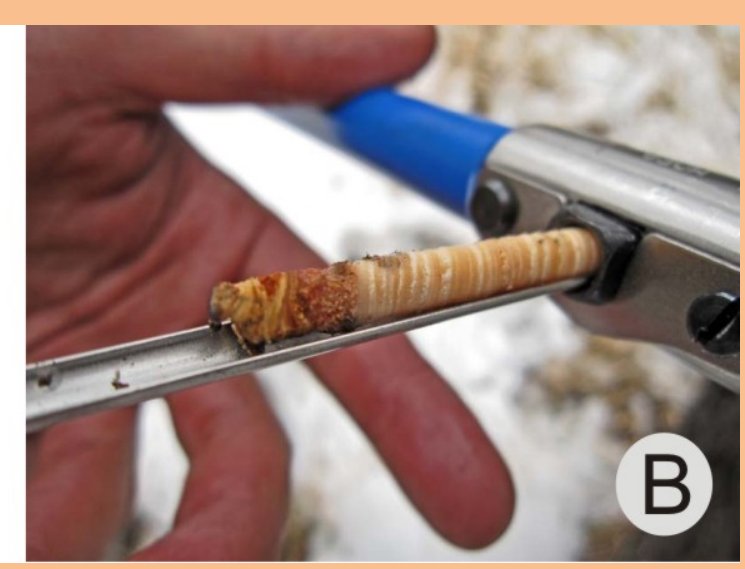

em que, Vol = volume de madeira visível na parte aérea da planta, $\mathrm{d}=$ diâmetro da circunferência do tronco dos indivíduos arbóreos, mensurados a altura do peito $(1,30 \mathrm{~m}), \mathrm{h}=$ altura da árvore $\mathrm{e} f \mathrm{f}=$ fator de forma, correspondente a relação entre o volume real do tronco de uma árvore pelo volume do cilindro da mesma altura e diâmetro da área basal, que no caso para a espécies em estudo o valor é de 0,51 para $E$. grandis (ALMEIDA et al., 2004) e 0,44 para $S$. jambos (WANG et al., 2009).

Figura 3. A) Estimativa da altura dos indivíduos arbóreos pelo método de comparação de referencial e B) mensuração do diâmetro do tronco com uma trena.

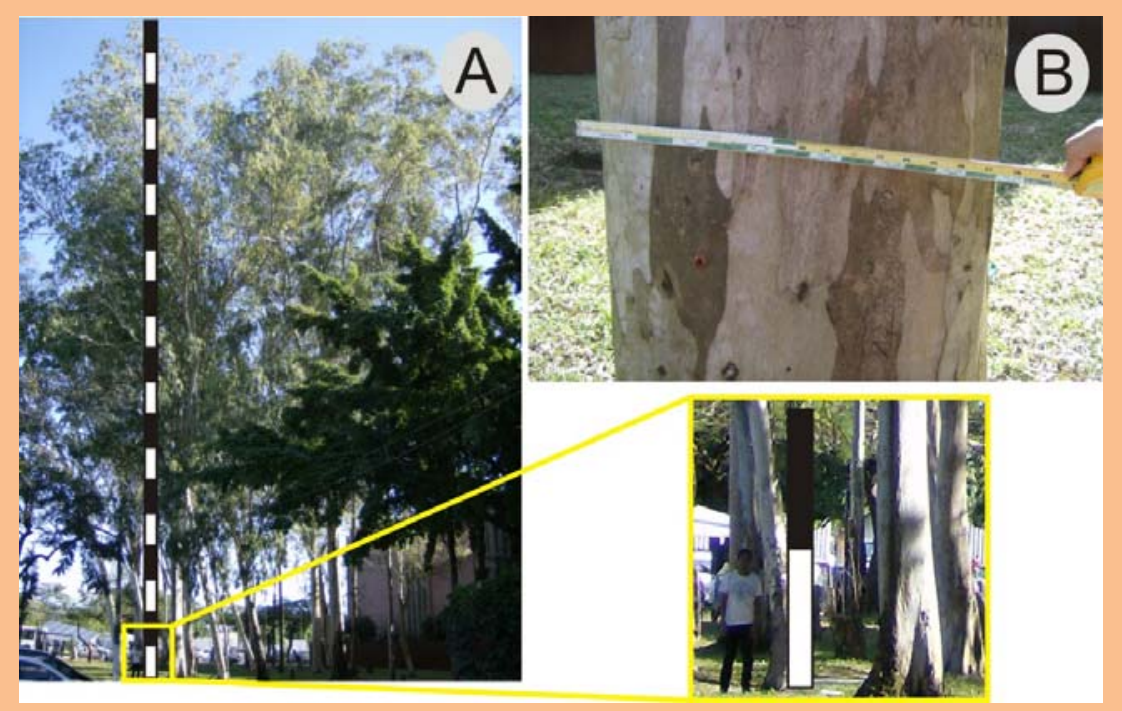

DIAGNÓSTICO DA IMPLANTAÇÃO DE Eucalyptus grandis... 
Teste de hipótese não paramétrico de MannWhitney foi efetuado para se calcular a diferença (valor de $\mathrm{p}=95 \%$ de confiabilidade) entre os grupos amostrais formados pelas espécies para a variável incremento médio anual dos anéis de crescimento. Análise de regressão foi efetuada entre a variável dependente (y) volume e independente $(\mathrm{x})$ idade para o cálculo do valor do coeficiente de determinação $=\mathrm{R}^{2}$; nível de significância $=\mathrm{p}$; equação e erro do ajuste dos dados a reta. Após isso foi aplicado o Teste de Chow, para calcular se há diferença entre as tendências de crescimento das curvas.

\section{RESULTADO E DISCUSSÃO}

Os indivíduos de $S$. jambos foram em média mais velhos do que os de E. grandis (Tabela 1, p < $0,0001)$, aproximadamente o dobro, e seguindo esta hierarquia de valor para outras variáveis mensuradas (Tabela 1, p $<0,0001$ ), sugerindo com isso a influência da idade nas variáveis dendrométricas.

Tabela 1. Média e desvio padrão das variáveis idade, diâmetro, altura e volume dos indivíduos das espécies estudadas no ano de 2011 (Agosto).

\begin{tabular}{lcccc}
\hline Espécies & Idade (anos) & DAP (cm) & Altura (m) & Volume $\left.\mathbf{( m}^{3}\right)$ \\
\hline Eucalyptus grandis & $19 \pm 3$ & $21,3 \pm 7,2$ & $31,1 \pm 2,3$ & $0,12 \pm 0,07$ \\
Syzygium jambos & $41 \pm 9$ & $27,1 \pm 11,1$ & $33,1 \pm 4,2$ & $0,25 \pm 0,10$ \\
\hline
\end{tabular}

$\mathrm{Na}$ análise dos anéis de crescimento dos últimos 10 anos (Figura 4A), foi observado que a espécie de $E$. grandis obteve um maior $(0,7 \pm 0,1)$ incremento no diâmetro do tronco do que a espécie $S$. jambos $(0,5$ $\pm 0,2 ; \mathrm{p}<0,001)$, confirmando as diferenças, Figura 4B (FERNÁNDEZ et al., 2011). Na Figura 4C, também foi observado nos últimos 10 anos através do incremento acumulado dos anéis de crescimento, que a espécie E. grandis apresentou uma angulação da reta maior, confirmando o rápido crescimento em relação à outra espécies no mesmo período de tempo. Uma hipótese para isto é que a espécie S. jambos, diferentemente do apresentado para espécie E. grandis, aloca seu crescimento na parte aérea e em uma parte não aérea, de raízes mais profundas e mais grossas do que a espécie $E$. grandis, que possui uma arquitetura de galhos e folhas ocupando uma maior área basal em relação a raízes profundas e frutos com mesocarpo desenvolvido (GUNARATNE et al., 2011).

A relação das idades de todos os indivíduos com o volume de madeira das espécies estudadas foram todas significativas $\left(\mathrm{R}^{2}>0,70 ; \mathrm{p}<0,015\right.$; Erro $<$ 0,05; Tabela 2) e houve uma relação diretamente significativa (Figura 5). Este resultado certificou o efeito que a idade nos valores de volume dos indivíduos mensurados e que a significância desta relação foi maior $\left(\mathrm{R}^{2}=0,82\right)$ na espécie $E$. grandis, comprovando que esta espécie cresce mais em um mesmo determinado do tempo. O resultado do Teste de Chow também foi significativo $(\mathrm{p}<0,001)$ mostrando que os conjuntos dos dados possuem tendências independentes e um destaque para o crescimento da espécie E. grandis, importante para Danilo Quintas Souto Maior et al. 
comprovar diferentes comportamentos ecofisiológicos das espécies (SCHWARZBAUM; KRUMSCHNABEL, 2011). Os resultados deste trabalho podem sugerir pela velocidade de crescimento observado entre as espécies, as diferentes estratégias dos indivíduos, corroborando com os dados da literatura que mostraram que cada espécie vegetal possui sua forma de investimento dos produtos metabólicos fotossintéticos (VALENTE et al. 2011; SHIMIZU; SPIR, 2004; ACERBI JR. et al., 2002).

Figura 4. A) Relação do incremento anual médio dos anéis de crescimento; B) Comparação das médias e desvios de todos os indivíduos entre as espécies, $\mathrm{p}$ = resultado do Teste não paramétrico de Mann-Whitney; e C) Incremento anual médio acumulado dos anéis de crescimento com o período de tempo avaliado (2001-2010).

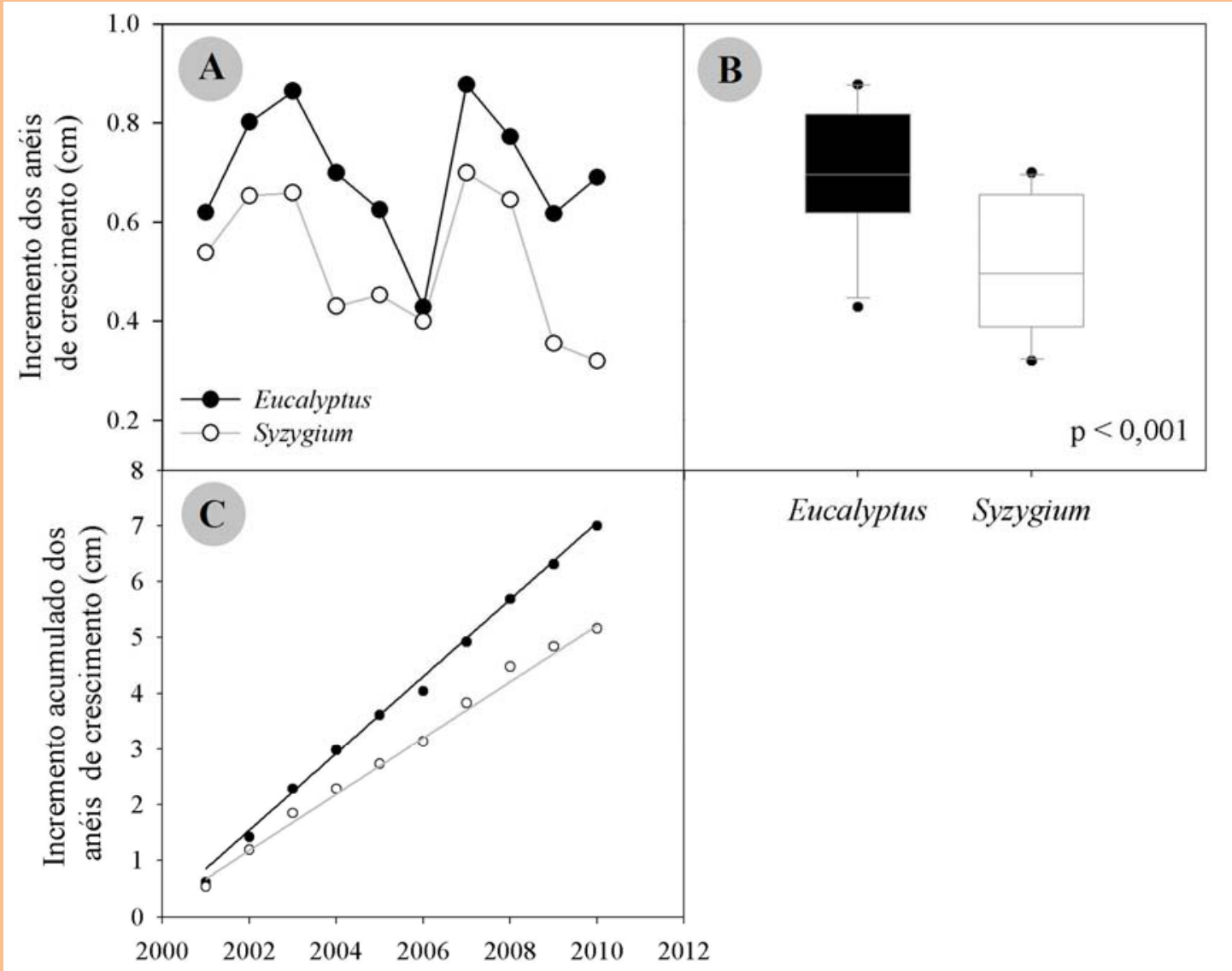

Tabela 2. Parâmetros da análise de regressão.

\begin{tabular}{lcccc}
\hline Espécie & $\mathbf{R}^{2}$ & $\mathbf{p}$ & Erro & Equação \\
\hline Eucalyptus grandis & 0,82 & 0,006 & 0,023 & $\mathrm{y}=0,01 \mathrm{x}-0,13+\varepsilon$ \\
Syzygium jambos & 0,76 & 0,014 & 0,031 & $\mathrm{y}=0,02 \mathrm{x}-0,24+\varepsilon$ \\
\hline
\end{tabular}


Figura 5. Relação entre volume e idade dos indivíduos arbóreos das duas espécies amostradas. $\mathrm{R}^{2}=$ coeficiente de determinação resultado da análise de regressão e $\mathrm{p}=$ nível de significância do Teste de Chow.

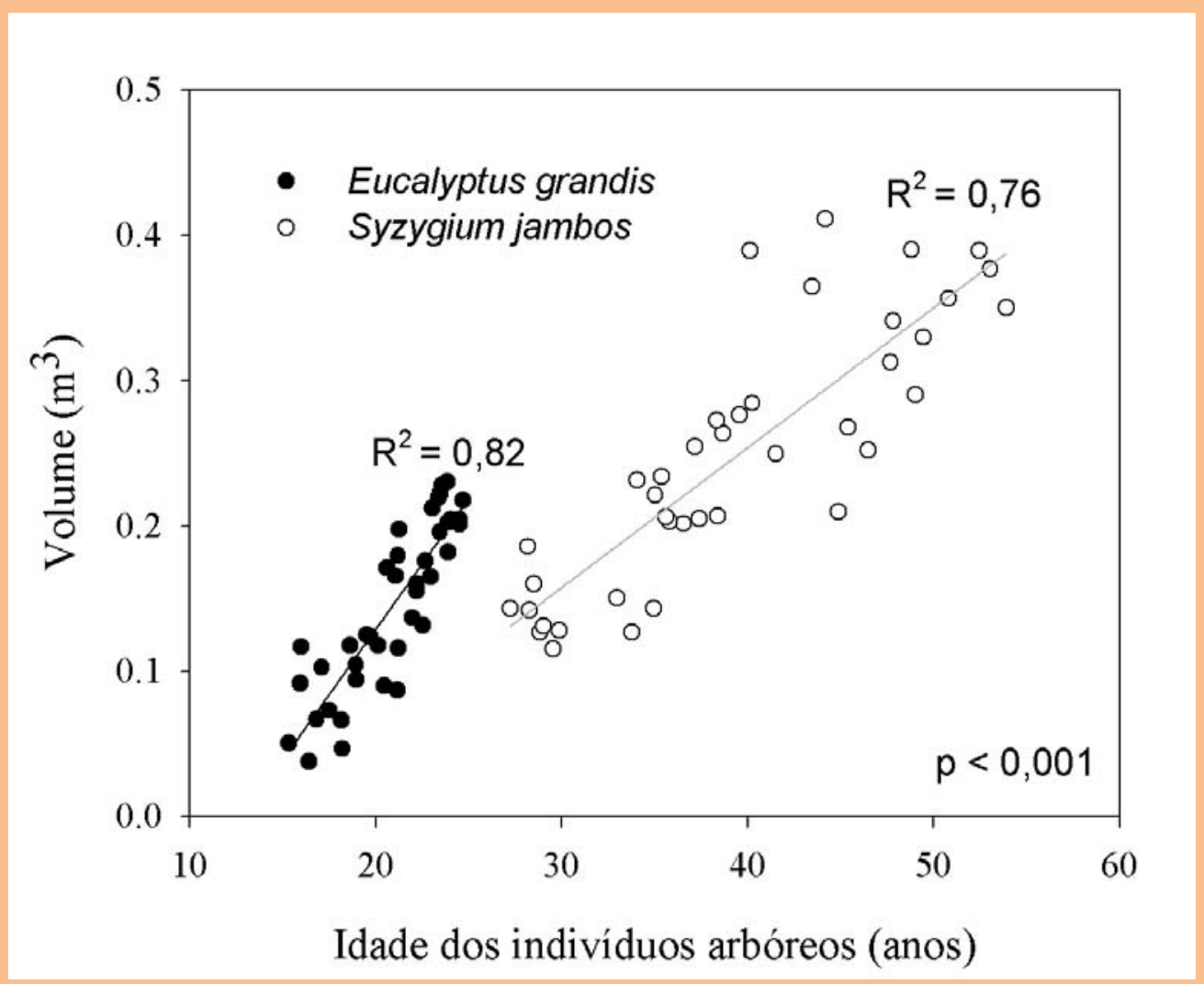

Observados os resultados, a sugestão das espécies na época atendia o Campus em questão nos objetivos propostos: sombreamento, quebra-vento, beleza cênica, porém com a expansão das redes e instalações elétricas e de comunicação (telefonia e internet) nos espaços verdes e nos caminhos para pedestre, tornaram inviável a sobrevivência conjunta dos indivíduos arbóreos com estas instalações urbanas. A expansão dos estacionamentos em áreas onde eram ocupadas pela espécie Syzygium jambos trouxeram alguns problemas, como, quedas de galhos e frutos sobre os automóveis. Observando a relação de causa e efeito, os espaços urbanos deveriam se adaptar ao planejamento arbóreo efetivado e não ao contrário
(JIM; ZHANG, 2013). Como isso não acontece, o desbaste e a erradicação de árvores introduzidas são frequentes em espaços urbanos (RINES et al., 2011). Atualmente, a implementação de espécies nativas são soluções imediatas para maximizar serviços ambientais, como biodiversidade, e por ser demorado seu crescimento frente as espécies introduzidas, retardam o aparecimento dos problemas de convivência entre as instalações e vias urbanas e os indivíduos arbóreos implementados (MOLLER; SKOU; KOLLMANN, 2012). Há no caso do Campus estudado, problemas de adaptação do que vai ser construído e expandido para preservação de indivíduos arbóreos. 
Os resultados mostram que a variável volume possui uma relação diretamente proporcional e significativa com a variável idade, sendo assim os indivíduos da espécie Eucalyptus grandis apresentaram um crescimento do tronco maior que Syzygium jambos, podendo ser devido ao modo de investimento de seus produtos fotossintéticos, conforme discutidos pela literatura. $\mathrm{O}$ crescimento dos indivíduos arbóreos no planejamento do
Campus da Universidade Federal de Pernambuco atenderam as expectativas propostas, porém, com a expansão das vias e das construções, os indivíduos arbóreos tornaram riscos a danos a estas instalações. A recomendação seria de que a expansão do Campus Universitário seguisse o planejamento arbóreo estabelecido incialmente, para não ter que no futuro realizar desbastes ou erradicação de indivíduos arbóreos.

\section{REFERÊNCIAS}

ACERBI JR., F. W. et al. Modelo para prognose do crescimento e da produção e análise econômica de regimes de manejo para Pinus taeda L. Rev. Árvore, v. 26, n. 6, p. 699-713, 2002.

ALMEIDA, A.C. et al. Needs and opportunities for using a process-based productivity model as a practical tool in Eucalyptus plantations. Forest Ecology and Management. v. 193, n. 1-2, p. 167-177, 2004.

ARIAS, D. et al. Productivity, aboveground biomass, nutrient uptake and carbon content in fast-growing tree plantations of native and introduced species in the Southern Region of Costa Rica. Original Biomass and Bioenergy. v. 35, n. 5, p. 1779-1788, 2011.

BACILIO, M.; VAZQUEZ, P.; BASHAN, Y. Water versus spacing: A possible growth preference among young individuals of the giant cardon cactus of the Baja California Peninsula. Environmental and Experimental Botany. v. 70, n. 1, p. 29-36, 2011.

BOULILA, W. et al. A data mining based approach to predict spatiotemporal changes in satellite images Original International. Journal of Applied Earth Observation and Geoinformation. v. 13, n. 3, p. 386-395, 2011.

BROWN, K.; COURTIN, P. J.; NEGRAVE, R. W. Growth, foliar nutrition and $\delta 13 \mathrm{C}$ responses of red alder (Alnus rubra) to phosphorus additions soon after planting on moist sites

Forest Ecology and Management. v. 262, n. 5, p. 791-802, 2011.

BRYAN, D. et al. Planting depth and soil amendments affect growth of Quercus virginiana Mill. Original Urban Forestry \& Urban Greening. v. 10, n. 2, p. 127-132, 2011.

CARNEIRO, M.F.H. et al. Pollen abortion rates, nitrogen dioxide by passive diffusive tubes and bioaccumulation in tree barks are effective in the characterization of air pollution. Environmental and Experimental Botany. v. 72, n. 2, p. 272-277, 2011.

COUTURIER, E.; PONT, S. C.; DOUADY, S. The filling law: A general framework for leaf folding and its consequences on leaf shape diversity. Journal of Theoretical Biology. v. 289, p. 47-64, 2011.

CRAVO, A.B. Frutas e ervas que curam: a panacéia vegetal. 4.ed. São Paulo: Hemus, 1994.

ENCINAS, J. I.; SILVA, G. F.; PINTO, J.R.R. Idade e crescimento das árvores. Brasília: Comunicações Técnicas Florestais, 2005 v. 7, n. 1.

FEIKEMA, P. M.; BAKER, T. G. Effect of soil salinity on growth of irrigated plantation Eucalyptus in southeastern Australia. Agricultural Water Management. v. 98, n. 7, p. 1180-1188, 2011.

FERNÁNDEZ, M. E. et al. Why do Pinus species have different growth dominance patterns than Eucalyptus 
species? A hypothesis based on differential physiological plasticity. Forest Ecology and Management, v. 261, n. 6, p. 1061-1068, 2011.

FRANZARING, J. et al. Growth, senescence and water use efficiency of spring oilseed rape (Brassica napus L. cv. Mozart) grown in a factorial combination of nitrogen supply and elevated $\mathrm{CO} 2$ Original. Environmental and Experimental Botany. v. 72, n. 2, p. 284-296, 2011.

GUNARATNE, A.M.T.A. et al. Release from root competition promotes tree seedling survival and growth following transplantation into human-induced grasslands in Sri Lanka. Forest Ecology and Management, v. 262, n. 2, p. 229-236, 2011.

HUTTON, R. J.; LOVEYS, B. R. A partial root zone drying irrigation strategy for citrus-Effects on water use efficiency and fruit characteristics. Agricultural Water Management. v. 98, n. 10, p. 1485-1496, 2011.

HYNYNEN, J.; REPOLA, J.; MIELIKÄINEN, K. The effects of species mixture on the growth and yield of mid-rotation mixed stands of Scots pine and silver birch Forest Ecology and Management. v. 262, n. 7, p. 1174-1183, 2011.

JIM, C.Y.; ZHANG, H. Species diversity and spatial differentiation of old-valuable trees in urban Hong Kong. Urban Forestry \& Urban Greening, v. 12, n. 2, p. 171-182, 2013.

KANTARC, M. D. Vertical climate zones in Biga peninsula: The impact of climate change and air pollution on forests. Procedia - Social and Behavioral Sciences. v. 19, p. 797-810, 2011.

KRUMM, F. et al. Stand development of Norway spruce dominated subalpine forests of the Swiss Alps. Forest Ecology and Management. v. 262, n. 4, p. 620-628, 2011.

MAXWELL, R. S.; WIXOM, J. A.; HESSL, A. E. A comparison of two techniques for measuring and crossdating tree rings. Dendrochronologia. v. 29, n. 4, p. 237-243, 2011.

MEYER, P.; SCHMIDT, M. Accumulation of dead wood in abandoned beech (Fagus sylvatica L.) forests in northwestern Germany. Forest Ecology and Management. v. 261, n. 3, p. 342-352, 2011.

MILLWARD, A. A.; SABIR, S. Benefits of a forested urban park: What is the value of Allan Gardens to the city of Toronto, Canada?. Landscape and Urban Planning. v. 100, n. 3, p. 177-188, 2011.

MOLlER, L. A.; SKOU, A. T.; KOLLMANN, J. Dispersal limitation at the expanding range margin of an evergreen tree in urban habitats? Urban Forestry \& Urban Greening, v. 11, n. 1, p. 59-64, 2012.

NASSER, N. UVB: suscetibilidade no melanoma maligno. An. Bras. Dermatol. v. 85, n. 6, p. 843-848, 2010.

PIECK, A. O. et al. Early seedling establishment of two tropical montane cloud forest tree species: The role of native and exotic grasses. Forest Ecology and Management. v. 261, n. 7, p. 1336-1343, 2011.

PIO CORREA, M. Dicionário das plantas úteis do Brasil e das exóticas cultivadas. Rio de Janeiro: Instituto Brasileiro de Desenvolvimento Florestal, 1969 v. 4, p. 427.

PCU - PREFEITURA DA CIDADE UNIVERSITÁRIA. Prefeitura da Cidade Universtária da Universidade Federal de Pernambuco (Campus Recife). Disponível em: <www.ufpe.br/pcu>. Acesso em Abril de 2013.

PRESTON, C. M. et al. The decomposition of windrowed, chipped logging slash and tree seedling response: A plant growth and nuclear magnetic resonance spectroscopy study. Organic Geochemistry. v. 42, n. 8, p, 936946, 2011.

RAMÍREZ, A. F. et al. Survival and growth of Acacia dealbata vs. native trees across an invasion front in southcentral Chile. Forest Ecology and Management. v. 261, n. 6, p. 1003-1009, 2011. 
RINES, D; et al. Measuring urban forestry performance and demographic associations in Massachusetts, USA. Urban Forestry \& Urban Greening, v. 10, n. 2, p. 113-118, 2011.

SCHWARZBAUM, P. J.; KRUMSCHNABEL,G. Perspective - from describing to understanding environmentphysiology relations: 50th birthday of a branch in ecophysiology. Comparative Biochemistry and Physiology Part A: Molecular \& Integrative Physiology, v. 158, n. 1, p. 9-12, 2011.

SEMENZATO, P.; CATTANEO, D.; DAINESE, M. Growth prediction for five tree species in an Italian urban forest. Original Research Article Urban Forestry \& Urban Greening. v. 10, n. 3, p. 169-176, 2011.

SHIMIZU et al. Produtividade de madeira de liquidambar (Liquidambar styraciflua L.) de diferentes procedências, em Quedas do Iguaçu, PR. Rev. Árvore. v. 28, n. 4, p. 487-491, 2004.

SILVA, M.L.; RIBEIRO, C.A.Á.S. Estabelecimento de rotação econômica para uma floresta regulada. Revista Árvore. v. 30, n. 1, p. 65-73, 2006.

SINGH, S. K.; RAI, J. P. N.; SINGH, A. Influence of prevailing disturbances on soil biology and biochemistry of montane habitats at Nanda Devi Biosphere Reserve (NDBR), India during wet and dry seasons. Geoderma. v. 162, n. 3-4, p. 296-302, 2011.

SLOWING, K.; CARRETERO, E.; VILLAR, A. Anti-inflammatory compounds of Eugenia jambos. Revista Phytother, p. 126-127, 1996.

SOARES, T.S.; CARVALHO, R.M.M.A.; VALE, A.B. Avaliação econômica de um povoamento de Eucalyptus grandis destinado a multiprodutos. Revista Árvore. v. 27, n. 5, p. 689-694, 2003.

SOUZA, C.R., ROSSI, L. M. B., AZEVEDO, C. P., LIMA, R. B. Comportamento da Acacia mangium e de clones de Eucalyptus grandis x Eucalyptus urophylla em plantios experimentais na Amazônia Central. Scientia Forestalis. v. 65, p. 95-101, 2004.

SREELATHA, S.; PADMA, P. R.; UMASANKARI, E. Evaluation of anticancer activity of ethanol extract of Sesbania grandiflora (Agati Sesban) against Ehrlich ascites carcinoma in Swiss albino mice. Journal of Ethnopharmacology. v. 134, n. 3, p. 984-987, 2011.

STRAW, N. et al. Defoliation and growth relationships for mid-rotation Sitka spruce attacked by the green spruce aphid, Elatobium abietinum (Walker) (Homoptera: Aphididae)

Forest Ecology and Management. v. 262, n. 7, p. 1223-1235, 2011.

UFNALSKI, K. A modified increment borer handle. Dendrochronologia. v. 23, n. 2, p. 105-106, 2005.

VALENTE, M. D. R. et al. Modelo de predição para o volume total de Quaruba (Vochysia inundata ducke) via análise de fatores e regressão. Rev. Árvore. v. 35, n. 2, p. 307-317, 2011.

WANG, W. et al. Development of TRIPLEX-Management model for simulating the response of forest growth to pre-commercial thinning. Ecological Modelling. v. 222, n. 14, p. 2249-2261, 2011.

WANG, Z. et al. Species-topography association in a species-rich subtropical forest of China. Basic and Applied Ecology. v. 10, n. 7, p. 648-655, 2009.

WU, D. et al. The CO2 Reduction Effects and Climate Benefit of Beijing 2008 Summer Olympics. Green Practice Energy Procedia. v. 5, p. 280-296, 2011.

YOSSIFOVA, M.; ESKENAZY, G. M.; VALCEVA, S. P. Petrology, mineralogy, and geochemistry of submarine coals and petrified forest in the Sozopol Bay, Bulgaria. International Journal of Coal Geology. v. 87, n. 3-4, p. 212-225, 2011.

ZHOU, X. et al. The use of forest-derived specific gravity for the conversion of volume to biomass for opengrown trees on agricultural land. Biomass and Bioenergy. v. 35, n. 5, p. 1721-1731, 2011.

DIAGNÓSTICO DA IMPLANTAÇÃO DE Eucalyptus grandis...

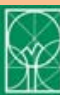

